\title{
PRODUÇÃO ARTESANAL ENTRE OS WAI-WAI EM ESPAÇOS DE UMA CASA DE APOIO A SAÚDE INDÍGENA
}

\author{
Nádile Juliane Costa de Castro ${ }^{1}$
}

Os povos indígenas possuem pelo Sistema Único de Saúde (SUS) o direito a serviços específicos da rede de atenção à saúde, entre eles polos administrativos e casas de apoio à saúde. No caso da etnia Wai-wai, estes recebem atendimento multiprofissional de saúde pela Casa de Apoio de Saúde Indígena de Oriximiná, município do oeste do Pará. São territórios, em geral, em um contexto biomédico, mas que se direcionam por práticas interculturais conforme preconizado pela Política Nacional de Atenção a Saúde dos Povos Indígenas.

Por certo, há uma necessidade de um o cuidado cultural nestes espaços, que é uma assistência a saúde pautada nas singularidades de povos, populações e comunidades locais considerando seus saberes, fazeres e realidades (LEININGER, 2001). A saúde é um traço cultural que está ligada a valores e concepções que fazem parte do seu constructo. Está baseada na cultura humana, e por tal, tem dentro do seu processo a formalização de instituições e organizações por onde as ações direcionadas são realizadas. Logo, o SUS, por exemplo, é uma instituição que influencia no cuidar e produzir saúde por meio de regras culturais técnicas ou tradicionais (CAMPOS, 2002).

A etnia Wai-wai possui a maior população no contexto de Oriximiná. Estes povos estão presentes principalmente na reserva Mapuera, que está localizada a margem esquerda do Rio Mapuera, com acesso por via fluvial através da cachoeira porteira, ou por via aérea, da sede municipal por aproximadamente 60 minutos.

A produção de seu artesanato é caracterizada pelo uso do Morototo (Schefflera morototoni). São colares, bolsas, instrumentos de origem xâmanica e adornos. O processo de aprendizagem do artesanato Wai-wai se dá através da oralidade. A confecção em geral é realizada por mulheres e repassadas para as gerações mais novas. A comercialização destes artefatos é um meio de renda, e são realizados diretamente pelo indígena ou por meio de lideranças da etnia. O processo de construção possui várias etapas: coleta do material (sementes naturais), preparação da matéria prima com coloração principalmente nas cores vermelha e roxa, além da cor natural da semente. Há também a implementação do processo com produtos como linhas de algodão e arame, para então ser finalizada a confecção.

\footnotetext{
${ }^{1}$ Doutora em Ciências Socioambientais pelo Núcleo de Altos Estudos Amazônicos da Universidade Federal do Pará. Mestre em doenças tropicais. Especialista em saúde pública. Graduada em Enfermagem. Pesquisa ensino, cultura e saúde de populações da Amazônia. E-mail: nadiledecastro@hotmail.com
} 
Este ensaio foi executado no ano 2018 e reúne um grupo de imagens que mostram a rotina diária dos povos da etnia Wai-wai na CASAI de Oriximiná, PA. As 10 imagens representam não somente os fazeres, mas o contexto da atenção aos povos indígenas da Amazônia como afirma Simonian (2007).

\section{REFERÊNCIAS}

CAMPOS, G. W. S. Sete considerações sobre saúde e cultura. Saúde e Sociedade, v. 11, p. 105-115, 2002.

LEININGER, M. Culture Care Diversity and Universality: a theory of Nursing. Boston: Jones and Barlett Pubs. XVI. 432p. 2001.

SIMONIAN, L.T.L. Uma relação que se amplia: fotografa e ciência sobre e na Amazônia. Imagens e pesquisas: ferramentas para compreensão da realidade amazônica. Belém: Editora do NAEA. p. 15-52; 2007.

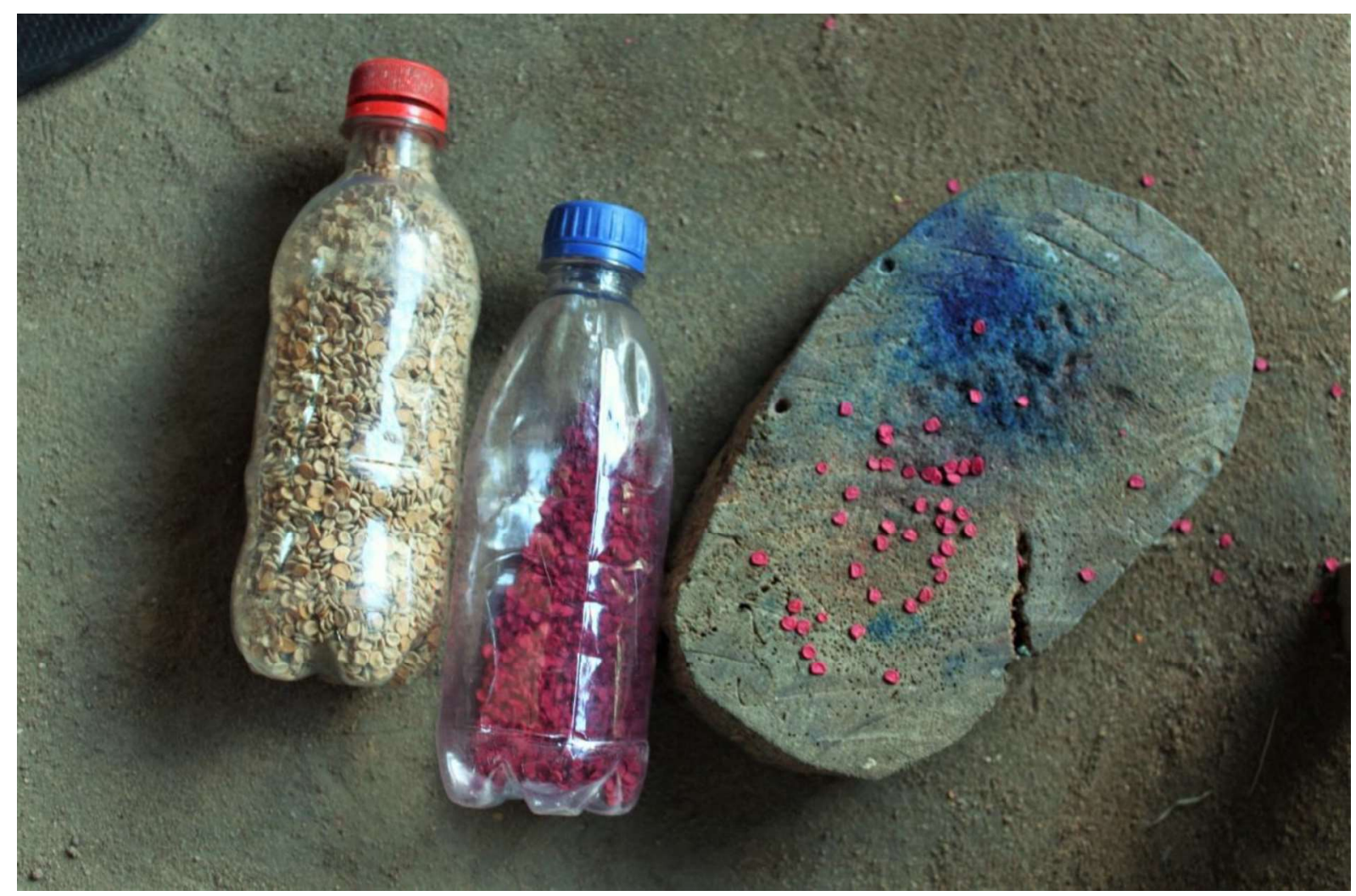



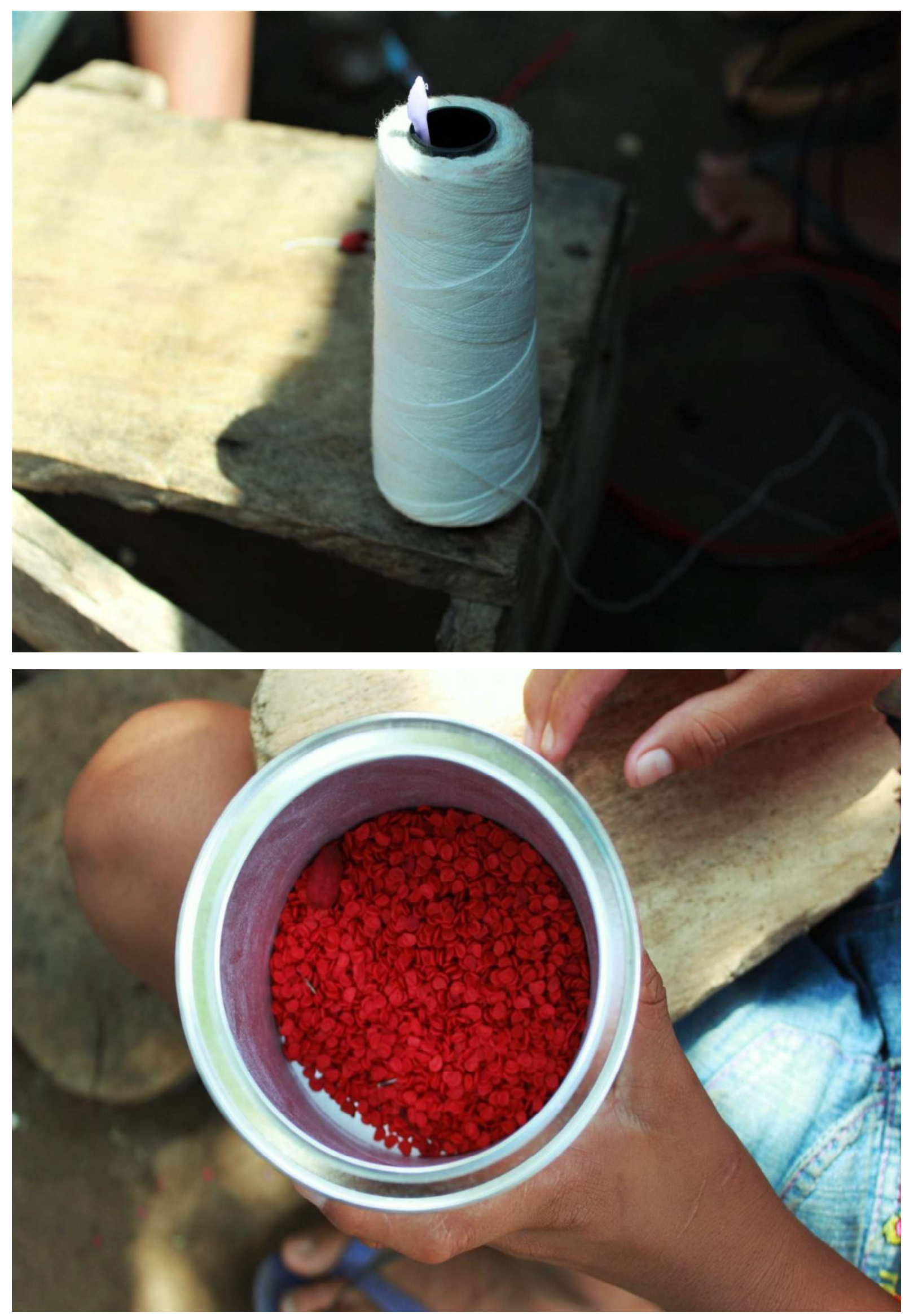

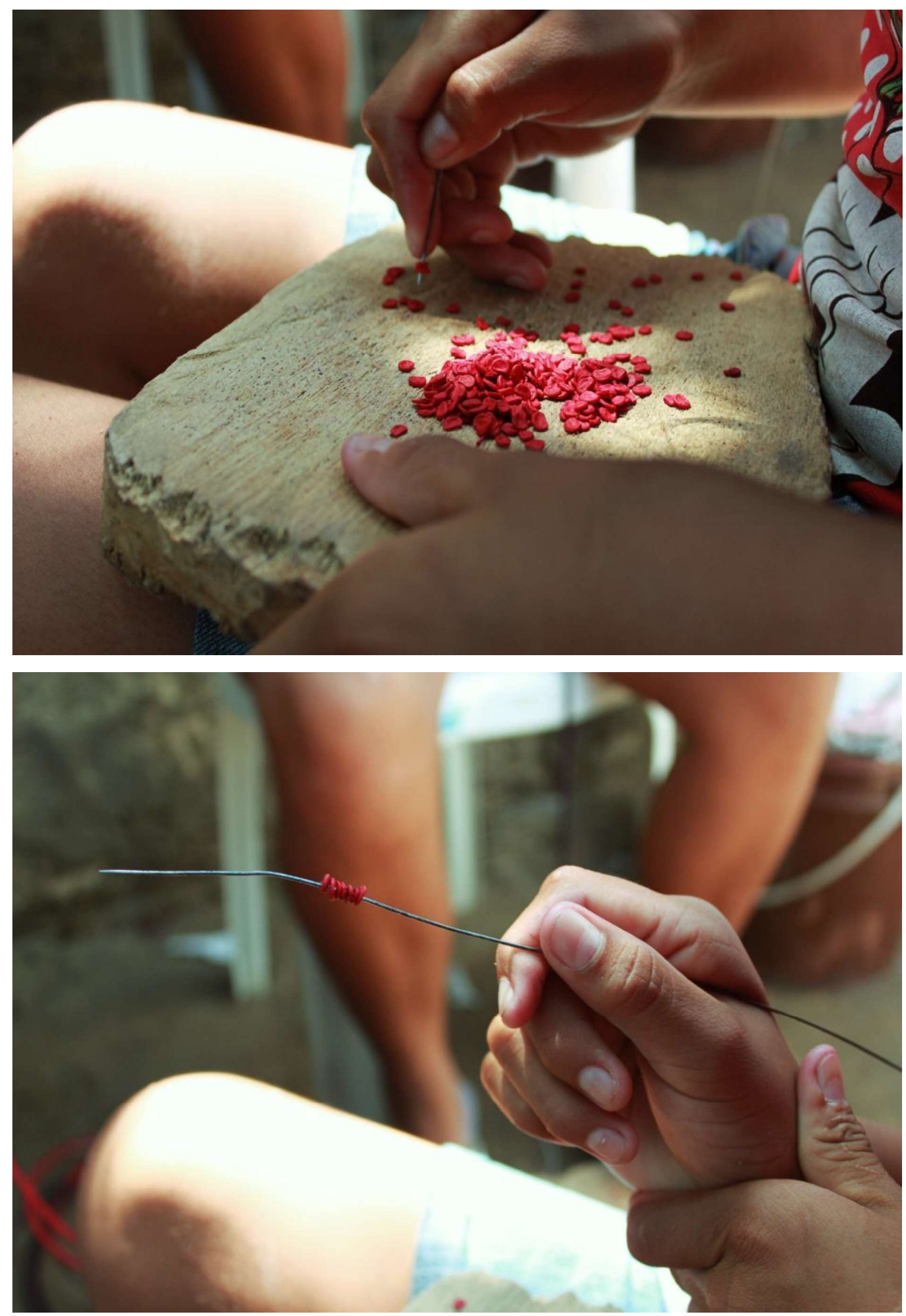

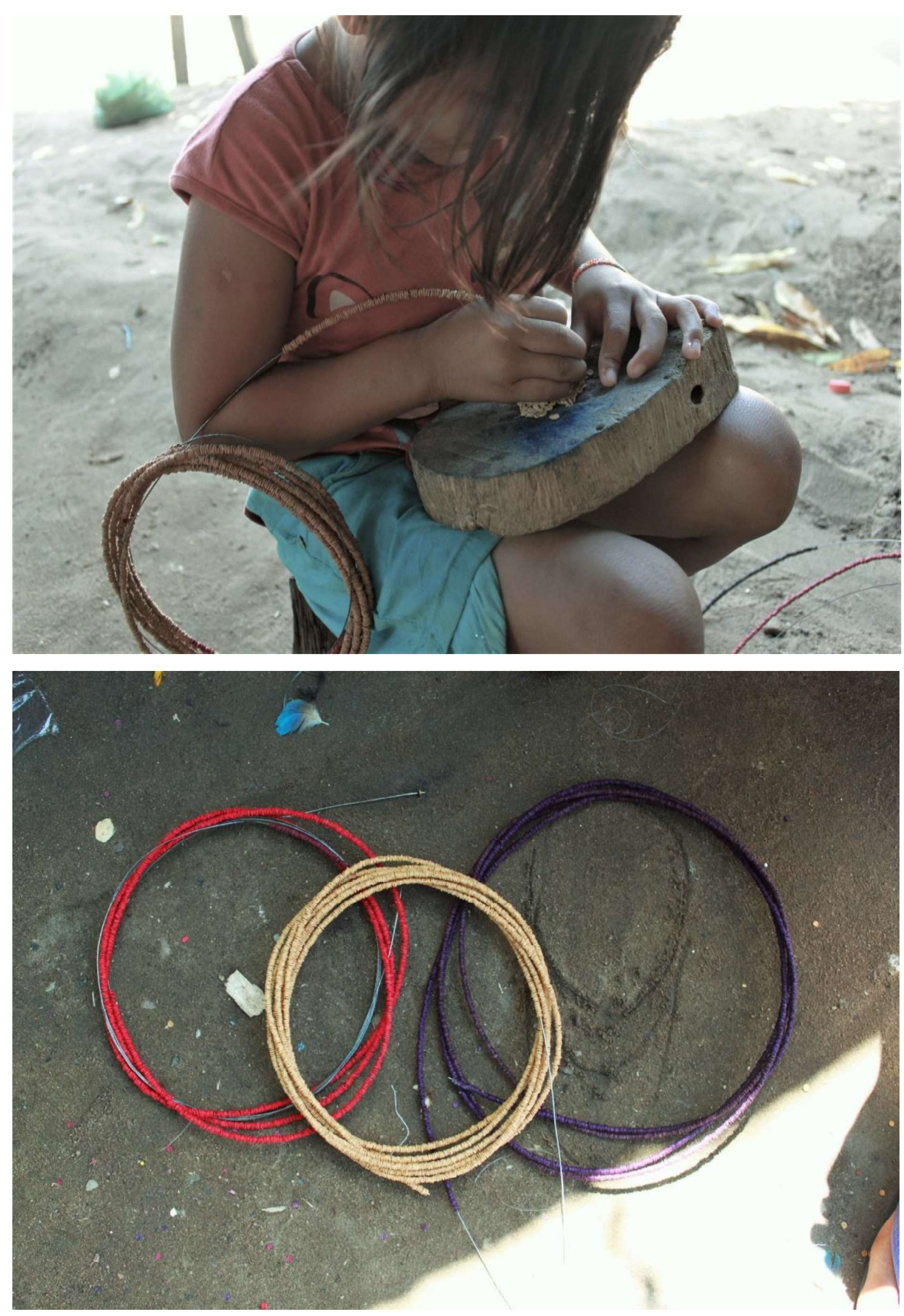

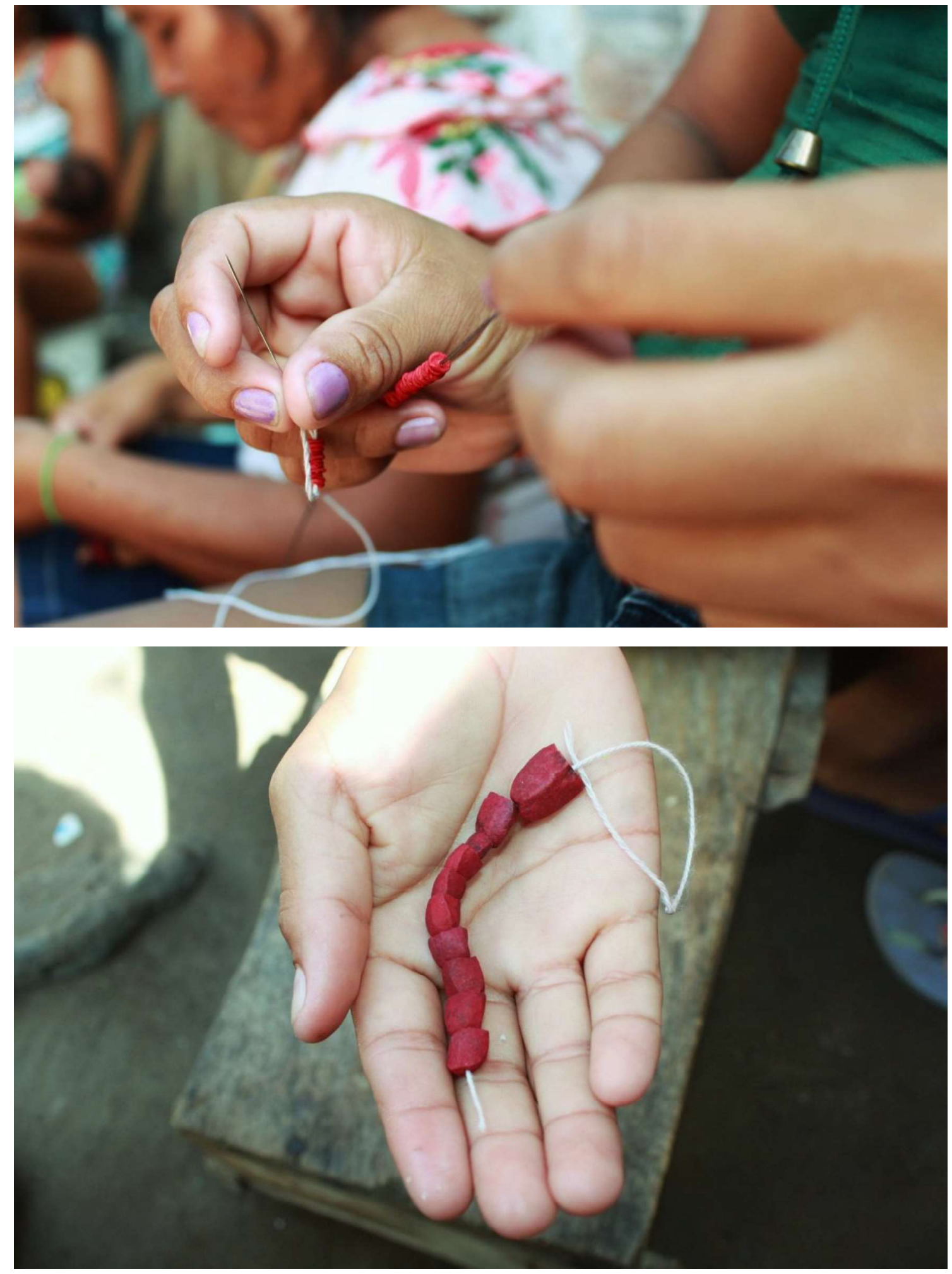


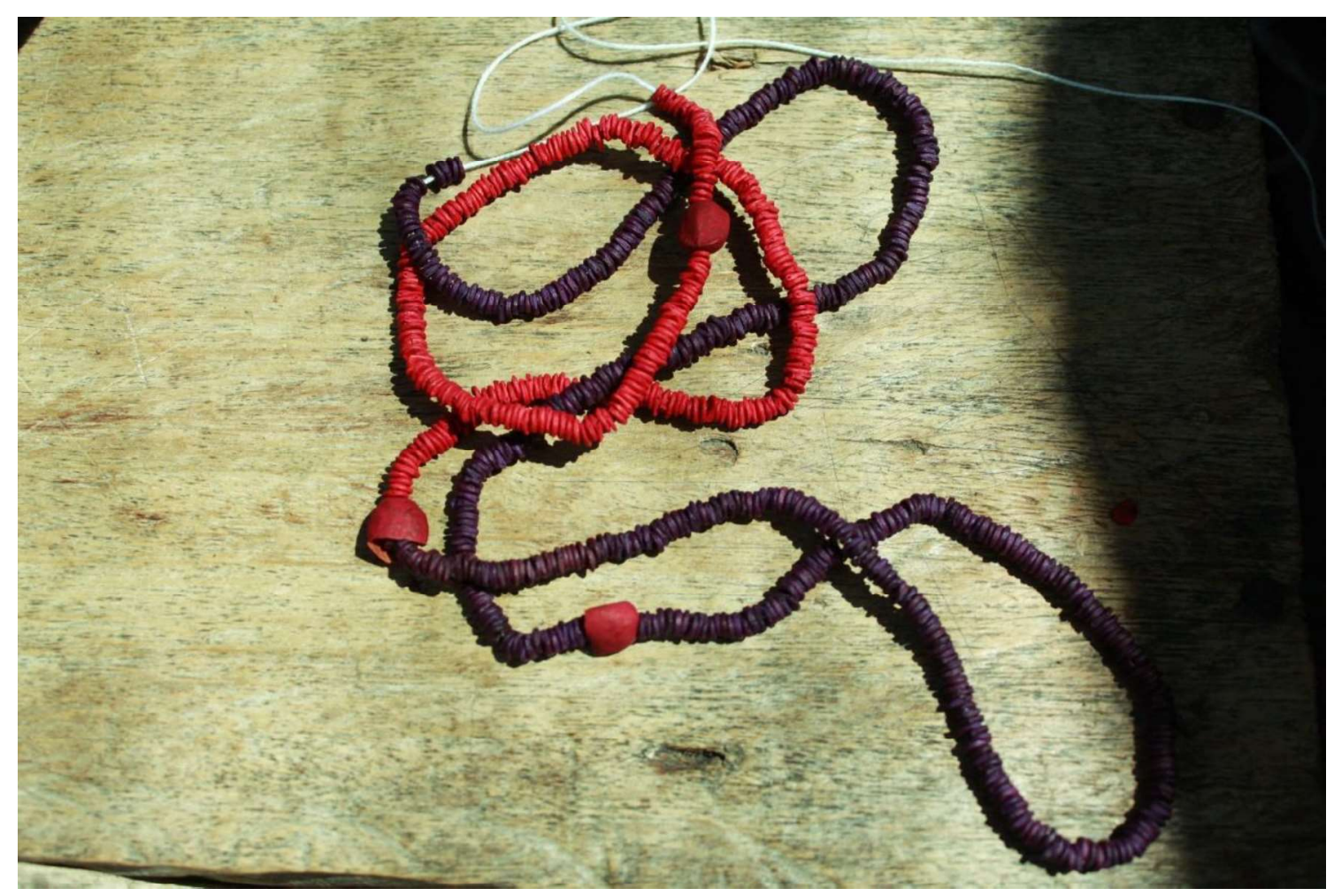

